\title{
Magnetic Resonance Imaging in the Diagnosis of Ankylosing Spondylitis: Be Aware of Gold Standards and Circularity
}

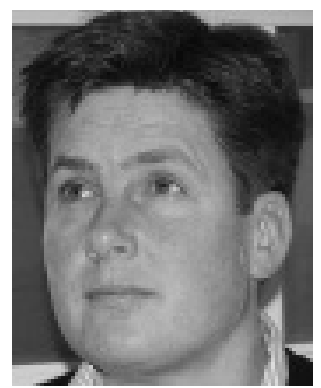

Magnetic resonance imaging (MRI) of the sacroiliac (SI) joints has rapidly gained attention as a potentially useful imaging modality in the diagnostic process of patients with chronic back pain. From a rheumatological point of view, MRI of the SI joints seems particularly useful in evaluating patients with inflammatory back pain (IBP), because it may help make or exclude a diagnosis belonging to the spectrum of spondyloarthritis (SpA). The prototype diagnosis in the spectrum of SpA obviously is ankylosing spondylitis (AS) or Bechterew's disease. It is useful to keep in mind that AS is a classifying diagnosis, based on clinical and radiological features articulated in the 1984 modified New York criteria ${ }^{1}$. The radiological criterion requires radiological sacroiliitis on a plain radiograph of the pelvis, and erosions belong to the features compatible with that. Two nuances should be mentioned here: The developers of the modified New York criteria investigated a group of classic AS patients, implying that many of these patients showed features that make AS classic, namely syndesmophytes or bamboo spine. This is why the modified New York criteria (and other sets of classification criteria in general) fail to detect patients with early disease and possibly even patients with mild or moderate disease. A second nuance is that the radiological criterion suffers from important interobserver variation, especially in the intermediate gradations of sacroiliitis ${ }^{2}$, implying that a classifying diagnosis of AS made by one clinician often does not hold up in the hands of another clinician. It is sometimes good to realize that we do not know exactly what AS is, especially when we talk about earlier or milder cases.

Some years ago, Rudwaleit, Khan, and Sieper recognized the above mentioned delay in diagnosing AS, saw MRI as a tool to fill the gap between start of complaints and diagnosis of AS, and have proposed the concept of axial $\mathrm{SpA}^{3}$. This concept states that there must be a pre-radiographic stage of AS that cannot yet be detected on radiographs but may be visualized by bone marrow edema (activity) on MRI of the SI joints, preceding structural (bony) changes that can be seen on radiographs. This concept has gained broad atten- tion, and there is some endorsing evidence in the literature ${ }^{4}$, but uncertainty remains whether all patients with early axial SpA develop into "full AS" and, if not, which factors determine such an outcome and what role do MRI abnormalities play. In order to facilitate studies that may shed light on this process, the Assessment of Spondyloarthritis International Society (ASAS) has endorsed and developed new criteria for axial $\mathrm{SpA}^{5,6}$, in which there is an important, although not dominant, role for MRI activity (not erosions) of the SI joints. Carefully conducted prospective studies in inception cohorts of patients presenting with signs of axial SpA will ultimately determine how useful MRI is in these patients with respect to patient prognosis: a classifying diagnosis of AS, syndesmophyte formation, or a chronic disease course.

In this issue of The Journal Wick, et $a l^{7}$ describe their experience with MRI abnormalities in patients with (a suspicion of) AS; they claim that it is erosions (chronic changes) rather than bone marrow edema or contrast medium enhancement (active changes) that distinguish AS from non-AS. Wick, et al have elegantly analyzed their data for which they should be commended, but this statement is too bold, may overestimate the value of erosions, and disqualify that of MRI activity.

My first conceptual remark is that it seems as if the authors have chased a contradictio in terminis by challenging the radiographic criterion of the modified New York criteria by MRI erosions. By default, it is impossible to beat the gold standard, even if the gold standard has inherent shortcomings as do the modified New York criteria. Obviously, and reassuringly, there is overlap between radiographic erosions and MRI erosions, which most likely explains why MRI erosions in this study are associated with a classification of AS. In analogy, this is why these patients have a higher C-reactive protein on average.

A second, more epidemiological concern, which is more generic too, pertains to the retrospective character of their study, which seriously hampers interpretability. The authors have retrospectively collected 179 patients with localized

See Erosions are the most relevant MRI features of SI quantification in AS, page 622

Personal non-commercial use only. The Journal of Rheumatology Copyright (c) 2010. All rights reserved. 
SI joint pain that sufficiently raised suspicion of AS to justify MRI examination. By doing so, they inherently created a selection of patients in whom (A) there was some suggestion of SI joint abnormality on clinical or radiological grounds; and (B) the pelvic radiograph was inconclusive. Patients with unambiguous pelvic radiographs (either unambiguously normal ór unambiguously abnormal) were not in this selection since MRI was considered redundant by the clinician (which may introduce left censorship bias), and the indication for performing the MRI was left to the discretion of the clinician (which may introduce indication bias, or confounding by indication). Both kinds of (potential) bias cannot be overcome in this retrospective design since it cannot be quantified, and in my opinion it is impossible to draw firm conclusions from such studies.

I will give one example to illustrate what could have happened: Suppose the authors had already "missed" 20 patients with classic AS showing unequivocal bilateral grade 3 sacroiliitis at presentation, so that these patients were not referred by their physician to MRI of the SI joints. Suppose these patients had shown erosions as well as clear bone marrow edema, which is not an unreasonable assumption in light of what we have shown in the Early SPondyloArthritis Cohort some years ago $^{8}$. That would mean that the proportion of patients with activity (bone marrow edema) versus those with chronicity (erosions) would markedly change, with potential implications for the conclusions of their study.

It is obvious that a lot of work still needs to be done to better understand how MRI could contribute in diagnosing patients belonging to the SpA spectrum and what prognostic information MRI can convey. In my opinion, such research should be prospective in nature, making sure that all patients presenting with a certain characteristic are captured (the design of an inception cohort), and should include patients with pre-radiographic disease, since only then it will become obvious how MRI abnormalities can contribute to a better diagnosis and, more importantly, to better prognostication.

In general, if we want to investigate the diagnostic or prognostic potential of a test, an assessment or an imaging tool, we should refrain from retrospective studies that can be contaminated by influences imposed by intangible external factors, such as physicians determining whether or not a particular test is required.

ROBERT B.M. LANDEWÉ, MD, Professor of Rheumatology, Maastricht University Medical Center and Caphri Research School, Maastricht, The Netherlands.

Address correspondence to Dr. R. Landewé, Maastricht University Medical Center, Department of Internal Medicine/Rheumatology, P.O. Box 5800, 6202AZ Maastricht, The Netherlands.E-mail r.landewe@mumc.nl

\section{REFERENCES}

1. van der Linden S, Valkenburg HA, Cats A. Evaluation of diagnostic criteria for ankylosing spondylitis. A proposal for modification of the New York criteria. Arthritis Rheum 1984;27:361-8.

2. Van Tubergen A, Heuft-Dorenbosch L, Schulpen G, Landewe R, Wijers R, Van Der Heijde D, et al. Radiographic assessment of sacroiliitis by radiologists and rheumatologists: does training improve quality? Ann Rheum Dis 2003;62:519-25.

3. Rudwaleit M, Khan MA, Sieper J. The challenge of diagnosis and classification in early ankylosing spondylitis: do we need new criteria? Arthritis Rheum 2005;52:1000-8.

4. Oostveen J, Prevo R, den Boer J, van de Laar M. Early detection of sacroiliitis on magnetic resonance imaging and subsequent development of sacroiliitis on plain radiography. A prospective, longitudinal study. J Rheumatol 1999;26:1953-8.

5. Rudwaleit M, Landewe R, van der Heijde D, Listing J, Brandt J, Braun J, et al. The development of Assessment of SpondyloArthritis international Society classification criteria for axial spondyloarthritis (part I): classification of paper patients by expert opinion including uncertainty appraisal. Ann Rheum Dis 2009;68:770-6.

6. Rudwaleit M, van der Heijde D, Landewe R, Listing J, Akkoc N, Brandt J, et al. The development of Assessment of SpondyloArthritis international Society classification criteria for axial spondyloarthritis (part II): validation and final selection. Ann Rheum Dis 2009;68:777-83.

7. Wick MC, Weiss RJ, Jaschke W, Klauser AS. Erosions are the most relevant magnetic resonance imaging features in quantification of sacroiliac joints in ankylosing spondylitis. J Rheumatol 2010;37:622-7.

8. Heuft-Dorenbosch L, Weijers R, Landewé R, van der Linden S, van der Heijde D. Magnetic resonance imaging changes of sacroiliac joints in patients with recent-onset inflammatory back pain: inter-reader reliability and prevalence of abnormalities. Arthritis Res Ther 2006;8:R11.

J Rheumatol 2010;37:477-8; doi:10.3899/jrheum.091396 